Relations industrielles

Industrial Relations

\title{
Conditions ouvrières et intégration sociale, par J. Maire Rainville, Éditions Ouvrières, Paris, 1967, 230 pp.
}

\section{Claude Charbonneau}

Volume 23, numéro 4, 1968

URI : https://id.erudit.org/iderudit/027961ar

DOI : https://doi.org/10.7202/027961ar

Aller au sommaire du numéro

Éditeur(s)

Département des relations industrielles de l'Université Laval

ISSN

0034-379X (imprimé)

1703-8138 (numérique)

Découvrir la revue

Citer ce compte rendu

Charbonneau, C. (1968). Compte rendu de [Conditions ouvrières et intégration sociale, par J. Maire Rainville, Éditions Ouvrières, Paris, 1967, 230 pp.] Relations industrielles / Industrial Relations, 23(4), 699-700.

https://doi.org/10.7202/027961ar

Tous droits réservés @ Département des relations industrielles de l'Université Laval, 1968
Ce document est protégé par la loi sur le droit d'auteur. L'utilisation des services d'Érudit (y compris la reproduction) est assujettie à sa politique d'utilisation que vous pouvez consulter en ligne.

https://apropos.erudit.org/fr/usagers/politique-dutilisation/ 


\section{RECENSIONS}

Le mouvement ouvrier aux Etats-Unis 1867-1967, par Daniel Guérin, FM/ Petite collection, Paris 1968, 176 pages.

Les options politiques et idéologiques de Daniel Guérin ne font aucun doute, elles sont clairement exposées dès l'avant-propos de ce petit ouvroge, où l'auteur se réfère ò Friedrich Engels et Isaac Deutscher. II est toujours intéressont de lire les écrits d'un européen, ò fortiori d'un intellectuel français de gouche sur la société américaine ou l'une de ses composantes; l'éloignement et les à priori idéologiques leur confèrent souvent un fumet bien porticulier.

Lo thèse de Daniel Guérin se résume fort simplement: le syndicalisme américain, Guérin dit le * Labor *, est un * géont endormi »; qu'il se réveille, il peut être - l'instrument décisif du socialisme ».

Si cet à priori, qui est également un pari, domine et oriente la démarche de l'auteur, celui-ci entend cependant respecter les faits et I'histoire, mais les perçoit à travers le prisme déformant de l'idéologie, et qui le conduit à surestimer, selon nous, les divergences existant dans la période octuelle entre les secteurs combatifs du « Labor * et les autres, c'est- $d$-dire entre certaines unions qui étaient affiliées à l'ancien $\mathrm{ClO}$ et d'autres qui l'étoient à la AFL. Ne faut-il pas, à tout prix, préserver le rôle et la mission historiques du mouvement et du syndicalisme ouvriers en tant qu'instrument de transformation de la société globale? Sinon I'homme de gauche rejoint inévitablement malgré toutes les nuances dont il fera étot la position d'Herbert Marcuse que, je cite ici Guérin * lors d'une rencontre d'intellectuels socialistes, d̀ New-York en septembre 1966... j'entendis affirmer d̀ l'encontre de l'enseignement du marxisme, que la classe ouvrière, dans les conditions du capitalisme avoncé, serait devenue anti-révolutionnaire, hostile au socialisme et qu'elle ne pourrait plus être considérée comme la force motrice du progrès

De tout ceci, nous tirerons deux conclusions: La première est une invitation non pcs à recuser les idéologies du moins dans la mesure où elles fournissent une problématique propre à faciliter la lecture des faits sociaux - prétendre ne pas en ovoir serait souscrire aux idées acquises - mais à les dépasser pour rejoindre au-delà des schémas et des jugements de valeur, qui les encombrent, le dynamisme et la densité des situations vécues.

La seconde est une invitation d̀ répondre à une interrogation fondamentale que nous serions tentés d'étendre au mouvement syndical conadien et qui se formulerait en ces termes: qu'en est-il aujourd'hui du rôle et de la volonté du mouvement syndical nordaméricain en tant qu'instrument de transformation de la société globale?

\section{Bernord SOLASSE}

\section{Conditions ouvrière et intégration sociale,} par J. Maire Rainville, Editions Ouvrières, Paris, 1967, 230 pp.

L'étude présentée vise d̀ connaitre a l'ajustement des ouvriers aux transformations sociales, économiques et culturelles de la société moderne ». Mais l'étude se limitant à des cas typiques ne donne qu'une image imparfaite des formes d'évolution en jeu.

Les principales variables ayant fait objet d'observations sont:

- La représentation au travail: L'intérêt au trovail ne se manifeste que lorsque tend à disparaitre l'état de subordination.

-La perception du statut social: Si l'inégalité de la condition ouvrière entraine l'affirmation des valeurs collectives, on voit aussi apparaître des niveaux d'aspirations individuelles au moyen de projets d'omélioration de niveau de vie.

-Lorientation des enfants: La plupart des ménages désirent la prolongation des études de leurs enfants et préfèrent pour eux une promotion en dehors de la voie personnellement suivie por eux.

- Les ressources et mode de vie: a) le confort familial en fonction des revenus indique une contradiction apparente entre logement vétuste et biens de consommation luxueux; b) acquis par le crédit qui amène les ménages à vivre au-dessus de leurs moyens, ce qui n'entrève pas la nécessité; c) d'autres privations pour préparer l'ovenir. 
- L'utilisation de l'espace urbain: ici l'auteur observe les comportements d'achat et les relations sociales qui demeurent ayant tout familiales. Les activités de loisirs ne sont pas envisagées comme moyens de promotion sociale: le but poursuivi demeure pour plusieurs la possession d'un confort familial. La mobilité géographique se limite ò la commune et au quartier.

Globalement l'étude montre que la tension entre deux modes de vie différents, l'un fondé sur les valeus traditionnelles d'entr'aide, de coopération; l'autre, fondé sur la consommation de masse, le profit, l'argent, n'a pas bouleversé l'ancien mode de vie à cause d'un manque de participotion des ouvriers dans le système.

\section{Claude CARBONNEAU}

L'emploi d temps partiel, Sixième rapport concernant l'emploi des groupes spéciaux, par Jean Hallaire, publié par la Direction de la Main-d'Oeuvre et des Affaires Sociales de I'O.C.D.E., Paris, 1968, 118 pp.

L'emploi à temps partiel o pris dans de nombreux pays de plus en plus d'importance. II répond aux besoins des travailleurs qui ne veulent ou ne peuvent travailler à plein temps.

L'auteur examine divers groupes de personnes pour lesquelles un emploi régulier à temps partiel semble approprié: les hommes qui ont des responsabilités familiales, les handicapés physiques, les étudiants, les personnes âgées, les veuves et divorcées et les femmes mariées. L'analyse repose sur l'expérience acquise dans six pays: Royaume-Uni, Allemagne, Suède, Danemark, Belgique, France, Etats-Unis et le Conada.

L'auteur passe ensuite en revue les arguments économiques et socioux en faveur du travail à temps partiel ainsi que ceux avancés por les organisations d'employeurs et de trovail contre l'emploi à temps partiel.

Les obstacles à l'emploi à temps partiel tels que la pression fiscale, l'inégalité des solaires entre hommes et femmes, et d'autres, sont analysés et des suggestions sont offertes pour les surmonter. Les garanties conventionnelles et réglementaires nécessaires à une meilleure intégration de ce type d'emploi dans le marché du travail sont étudiées.

Ce ropport fait des prévisions quant ò la place future de l'emploi à temps partiel, notamment pour les groupes d'activités suivants: infirmières, secteur public et paro- public (I'enseignement), l'industrie, le secteur tertiaire: en particulier les octivités de loisirs.

Les conclusions et recommandations de l'outeur intéresseront tous les services gouvernementaux, les associations d'employeurs et de travoilleurs qui cherchent des solutions aux problèmes soulevés par l'emploi d temps partiel.

\section{Pierre DIONNE}

La mobilité des travailleurs urbains, par Laurence C. Hunter et Graham L. Reid, O.C.D.E., Paris, 1968, 239 pp.

Cette étude complète la liste des publications approuvées par le comité de la maind'oeuvre et des affaires sociales de l'organisation de coopération et de développement économiques, dont l'intérêt justifie que nous roppelions ici les titres:

$H$. KRIER, Main-d'oeurre rurale et le développement industriel, O.C.D.E.

G. BARBICHON, Adaptation ef formation de la main-d'oeurre des régions rurales au travail industriel, O.C.D.E.

G. BEIJER, La main-d'oeuvre rurale nationale, son adaptation à l'industrie, O.C.D.E

R. DESCLOITRES, Le travailleur étranger ; son adaptation au travail industriel et ò la vie urbaine, O.C.D.E.

Dons leur étude MM. Hunter et Reid érigent en principe que la ropidité du progrès technologique et, son corrélat, l'évolution des structures de l'emploi exigent un occroissement de la mobilité de la main-d'oeuvre. Ce raisonnement les conduit ò légitimer une intervention des pouvoirs publics destinée d̀ inciter les travailleurs à délaisser volontairement les régions et les industries en déclin au profit de régions et $d$ 'industries en expansion.

Certes ces auteurs n'ignorent pas les facteurs de résistance "non économiques * propres à freiner la mobilité géographique des travailleurs, mais semblent les sousestimer quelque peu. C'est là l'une des limites logiques d'une opproche trop exclusivement d'ordre économique.

Les conclusions, qui ne sont en fait que des recommandations à peine voilées, nous paraissent trop générales pour pouvoir servir de base à l'élaboration de politiques gouvernementales effectives, tant il est vrai qu'en cette matière, les motivotions et les décisions des individus dans un contexte et des situa- 